\title{
Viscous Droplet Impact on Non-wettable Textured Surfaces
}

Mehran Abolghasemibizaki ${ }^{\mathrm{a}, \mathrm{b}}$, Neda Dilmaghani ${ }^{\mathrm{a}}$, Reza Mohammadi ${ }^{\mathrm{a}^{*}}$, and Carlos E. Castano ${ }^{\mathrm{a}^{*}}$

a Department of Mechanical and Nuclear Engineering, Virginia Commonwealth University, Richmond, VA, 23284, USA

${ }^{\mathrm{b}}$ Department of Mechanical Engineering, Johns Hopkins University, Baltimore, MD, 21218, USA

*Corresponding Authors: rmohammadi@vcu.edu; Phone: (804)827-3997. cecastanolond@,vcu.edu; Phone: (804)828-1998.

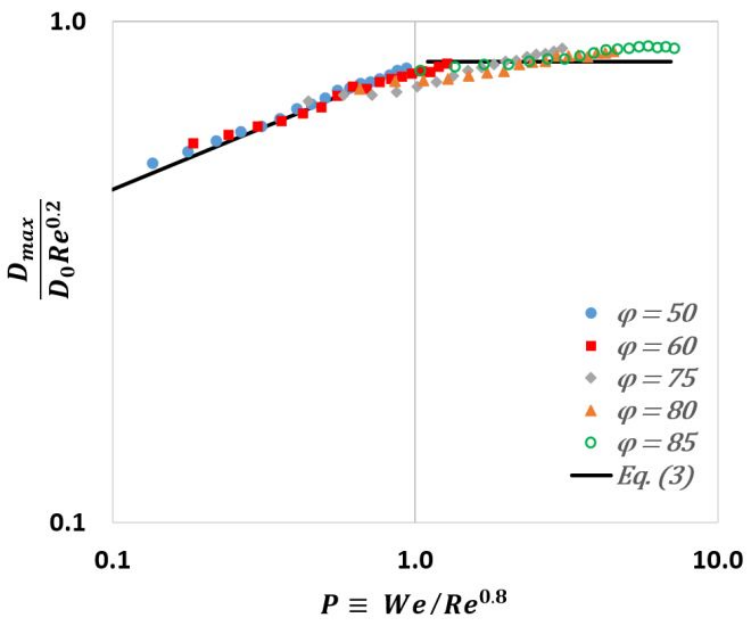

Figure S1. Variation of maximum spreading diameter $\left(D_{\max }\right)$ for the impacting drops with respect to the Impact number. This graph is presented only to express more details for Figure 4 of the manuscript. It can be seen the most discrepancy between Eq. 3 and the data happens in the vicinity of $P=1$, which can be neglected. 


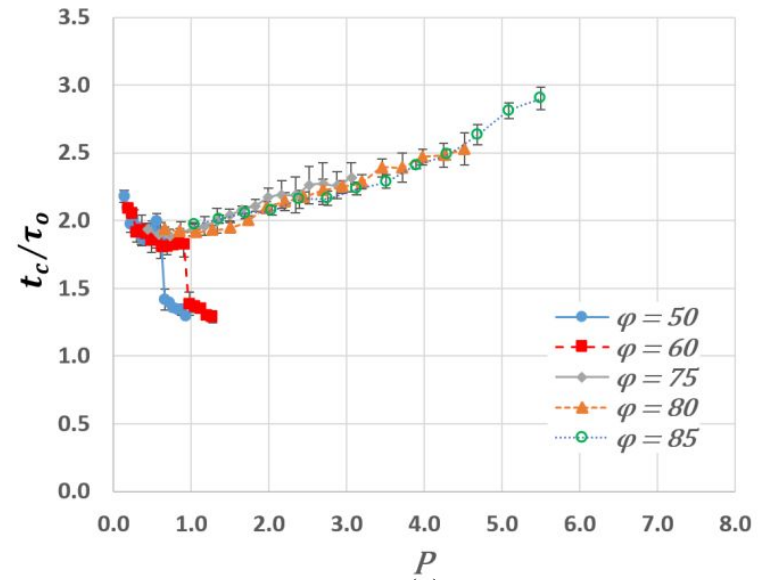

(a)

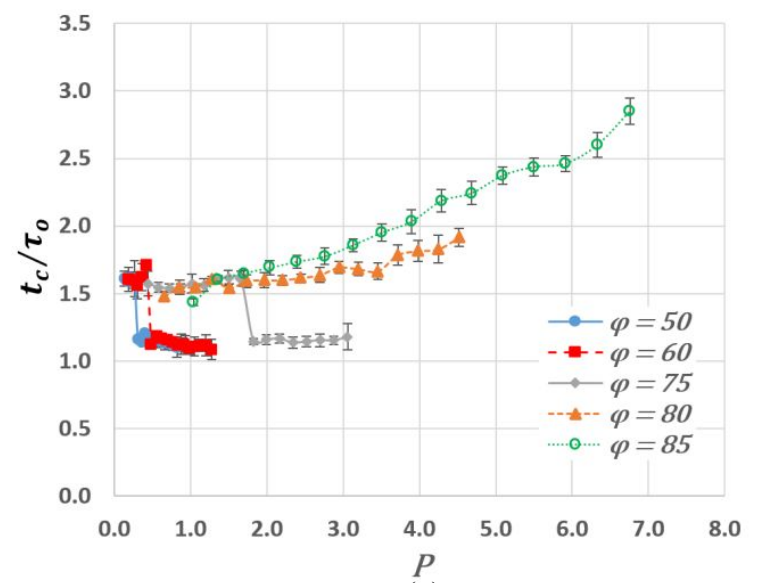

(c)

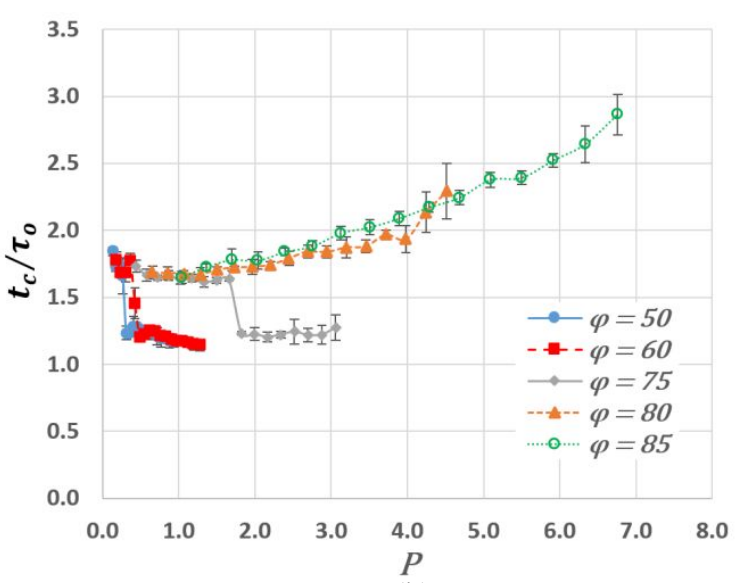

(b)

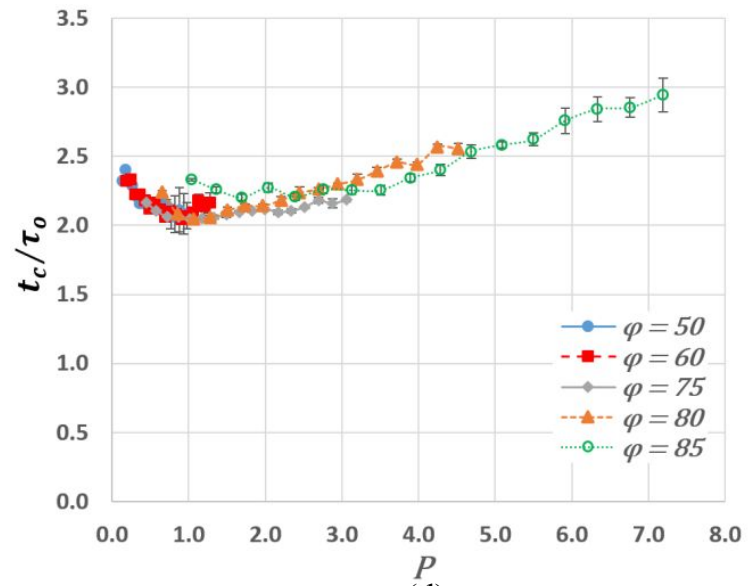

(d)

Figure S2. The plots depict the variation of the contact time of glycerol-water mixtures (different $\varphi)$ normalized by inertial-capillary time scale $\left(\tau_{o}=\sqrt{\rho D_{o}^{3} / 8 \gamma}\right)$ with respect to the Impact number $\left(P \equiv \sum_{e^{\prime}} e^{\frac{4}{5}}\right)$ for surfaces with macroscopic wire of (a) $d=0.1$, (b) $d=0.3$, and (c) $d=$ $0.5 \mathrm{~mm}$. (d) Plot shows the same variation for the ribbed surface; the trend is similar to that of the smooth surface with shorter contact time. 


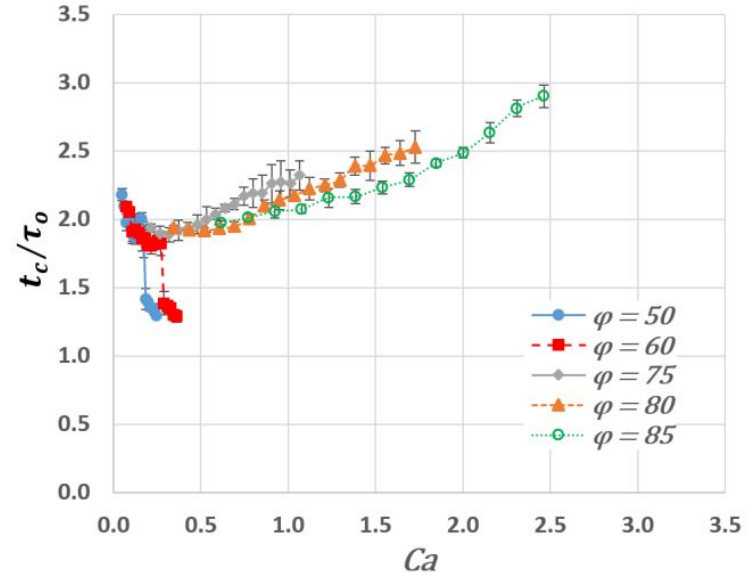

(a)

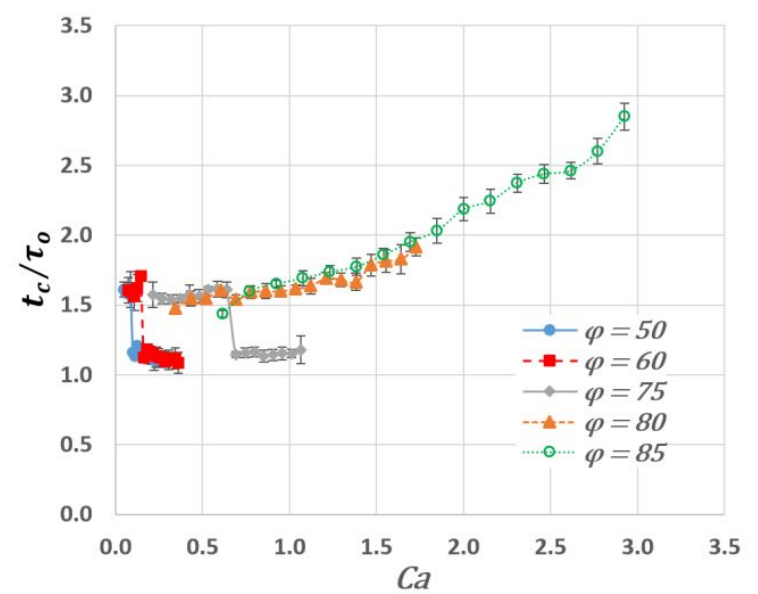

(c)

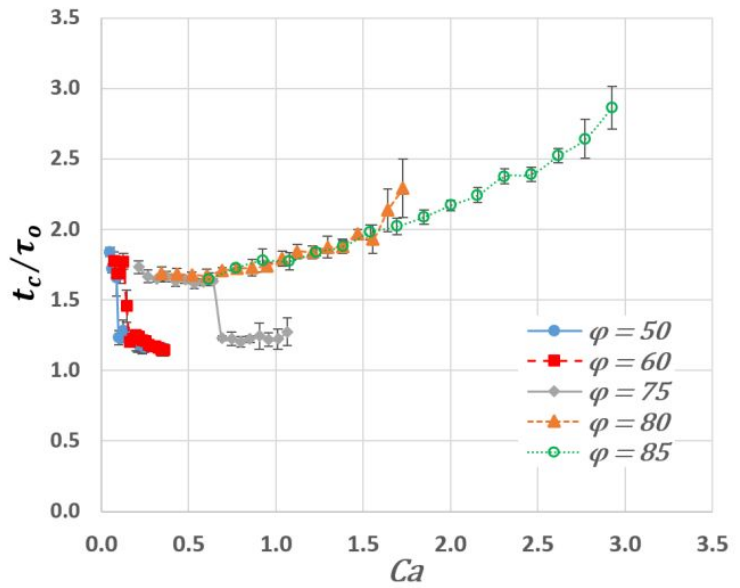

(b)

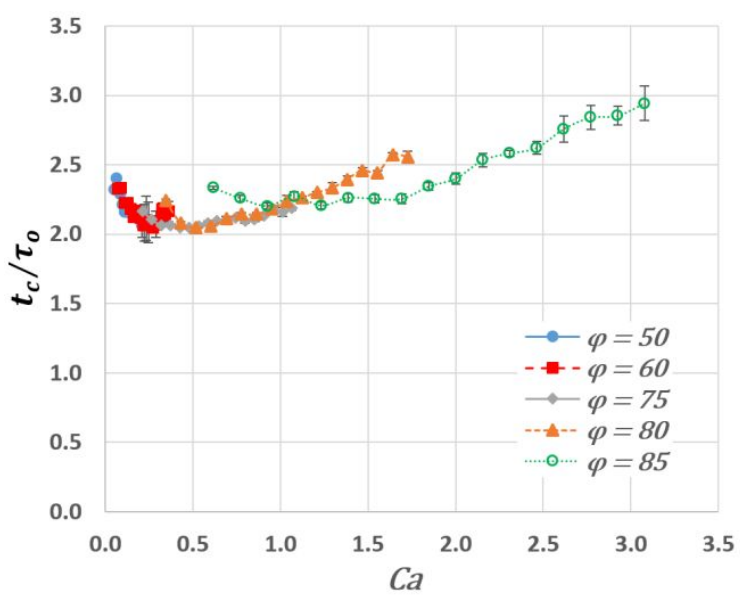

(d)

Figure S3. Normalized contact time for the impacts of various Capillary number on the smooth surfaces with wire of (a) $d=0.1$, (b) $d=0.3$, and (c) $d=0.5 \mathrm{~mm}$ as well as (d) the ribbed surface. While critical Capillary numbers of 2.5 (for $d=0.1 \mathrm{~mm}$ ) and 3.0 (for $d=0.3$ and $0.5 \mathrm{~mm}$ ) were found for wired surfaces, beyond which no detachment from the surface happens, drop detachment from ribbed surface was observed for all the conditions tested in this work. 